\title{
Orexin in the anxiety spectrum: association of a HCRTR1 polymorphism with panic disorder/agoraphobia, CBT treatment response and fear-related intermediate phenotypes
}

Michael G. Gottschalk¹,2, Jan Richter ${ }^{3}$, Christiane Ziegler $\mathbb{D}^{1}$, Miriam A. Schiele ${ }^{1}$, Julia Mann², Maximilian J. Geiger ${ }^{2,4}$, Christoph Schartner $\mathbb{0}^{2,5}$, György A. Homola ${ }^{6}$, Georg W. Alpers ${ }^{7}$, Christian Büchel ${ }^{8}$, Lydia Fehm', Thomas Fydrich ${ }^{9}$, Alexander L. Gerlach $\mathbb{1 0}^{10}$, Andrew T. Gloster ${ }^{11,12}$, Sylvia Helbig-Lang ${ }^{11,13}$, Raffael Kalisch ${ }^{14}$, Tilo Kircher $^{15}$, Thomas Lang ${ }^{11,13,16}$, Tina B. Lonsdorf ${ }^{8}$, Christiane A. Pané-Farré ${ }^{3}$, Andreas Ströhle ${ }^{17}$, Heike Weber $\mathbb{B}^{2,18}$, Peter Zwanzger 19,20,21, Volker Arolt ${ }^{19}$, Marcel Romanos ${ }^{22}$, Hans-Ulrich Wittchen ${ }^{11,21}$, Alfons Hamm³ , Paul Pauli $\mathbb{C}^{23}$, Andreas Reif $\mathbb{0}^{18}$, Jürgen Deckert ${ }^{2}$, Susanne Neufang ${ }^{22,24}$, Michael Höfler ${ }^{11}$ and Katharina Domschke ${ }^{1,25}$

\begin{abstract}
Preclinical studies point to a pivotal role of the orexin $1\left(\mathrm{OX}_{1}\right)$ receptor in arousal and fear learning and therefore suggest the HCRTR1 gene as a prime candidate in panic disorder (PD) with/without agoraphobia (AG), PD/AG treatment response, and PD/AG-related intermediate phenotypes. Here, a multilevel approach was applied to test the non-synonymous HCRTR1 C/T lle408Val gene variant (rs2271933) for association with PD/AG in two independent casecontrol samples (total $n=613$ cases, 1839 healthy subjects), as an outcome predictor of a six-weeks exposure-based cognitive behavioral therapy (CBT) in PD/AG patients $(n=189)$, as well as with respect to agoraphobic cognitions (ACQ) ( $n=483$ patients, $n=2382$ healthy subjects), fMRI alerting network activation in healthy subjects $(n=94)$, and a behavioral avoidance task in PD/AG pre- and post-CBT $(n=271)$. The HCRTR1 rs2271933 T allele was associated with $\mathrm{PD} / \mathrm{AG}$ in both samples independently, and in their meta-analysis $\left(p=4.2 \times 10^{-7}\right)$, particularly in the female subsample $\left(p=9.8 \times 10^{-9}\right)$. T allele carriers displayed a significantly poorer CBT outcome (e.g., Hamilton anxiety rating scale: $p=$ $7.5 \times 10^{-4}$ ). The T allele count was linked to higher ACQ sores in PD/AG and healthy subjects, decreased inferior frontal gyrus and increased locus coeruleus activation in the alerting network. Finally, the $T$ allele count was associated with increased pre-CBT exposure avoidance and autonomic arousal as well as decreased post-CBT improvement. In sum, the present results provide converging evidence for an involvement of HCRTR1 gene variation in the etiology of PD/ $A G$ and PD/AG-related traits as well as treatment response to CBT, supporting future therapeutic approaches targeting the orexin-related arousal system.
\end{abstract}

\footnotetext{
Correspondence: Katharina Domschke (katharina.domschke@uniklinik-freiburg.

de)

${ }^{1}$ Department of Psychiatry and Psychotherapy, Medical Center - University of Freiburg, Faculty of Medicine, University of Freiburg, Freiburg, Germany

${ }^{2}$ Department of Psychiatry, Psychosomatics and Psychotherapy, Center of Mental Health, University Hospital of Würzburg, Würzburg, Germany

Full list of author information is available at the end of the article.

Shared first authors: Michael G. Gottschalk, Jan Richter, Christiane Ziegler

Shared last authors: Susanne Neufang, Michael Höfler, Katharina Domschke
}

\section{Introduction}

The general term orexin (or hypocretin) refers to the two known hypothalamic neuropeptides comprised in the orexin class, namely orexin-A and -B (hypocretin $1 / 2)^{1}$. The orexin system has been implicated in the

\section{(c) The Author(s) 2019}

(c) (i) Open Access This article is licensed under a Creative Commons Attribution 4.0 International License, which permits use, sharing, adaptation, distribution and reproduction cc) in any medium or format, as long as you give appropriate credit to the original author(s) and the source, provide a link to the Creative Commons license, and indicate if changes were made. The images or other third party material in this article are included in the article's Creative Commons license, unless indicated otherwise in a credit line to the material. If material is not included in the article's Creative Commons license and your intended use is not permitted by statutory regulation or exceeds the permitted use, you will need to obtain permission directly from the copyright holder. To view a copy of this license, visit http://creativecommons.org/licenses/by/4.0/. 
maintenance of arousal, wakefulness, and vigilance as well as in influencing motivated behaviors such as emotional responses, reward seeking and feeding ${ }^{2}$.

The action of orexin- $A$ and $-\mathrm{B}$ is mediated by two subtypes of G-protein coupled receptors, the orexin 1 $\left(\mathrm{OX}_{1}\right)$ receptor and the orexin $2\left(\mathrm{OX}_{2}\right)$ receptor $^{3,4}$. While both receptors are co-expressed in the dorsal raphe nuclei and ventral tegmental area, the $\mathrm{OX}_{1}$ and $\mathrm{OX}_{2}$ receptors display characteristic anatomical distributions, suggesting distinct physiological functions based on specific neuronal signaling pathways: the $\mathrm{OX}_{1}$ receptor is primarily expressed in the locus coeruleus (LC), laterodorsal and pedunculopontine tegmental nucleus, while the $\mathrm{OX}_{2}$ receptor is located in the arcuate and tuberomammillary nucleus ${ }^{5}$. In addition, site-specific exclusive expression of the $\mathrm{OX}_{1}$ receptor has been reported in the cingulate cortex, CA1/2 region of the hippocampus, bed nucleus of the stria terminalis and amygdala ${ }^{5}$. Nonetheless, it should be kept in mind that gene expression information is ultimately limited by mRNA detection and antibody staining sensitivity and specificity.

Besides this abundance of the $\mathrm{OX}_{1}$ receptor in circuity engaged during fear ${ }^{6}$, a pivotal role of the $\mathrm{OX}_{1}$ receptor in the pathogenesis of hyperarousal- and panic-related anxiety has been suggested by both preclinical and clinical studies: ${ }^{7}$ In a rodent model, the orexin system has been shown to modulate the formation and expression of fear memory via noradrenergic neurons in the LC expressing the $\mathrm{OX}_{1}$ receptor, with $H \operatorname{crtrl}(-/-)$ mice displaying impaired freezing responses in both cued and contextual fear-conditioning paradigms ${ }^{8}$. Additionally, blocking orexin activity in the noradrenergic LC has been reported to reduce fear learning in a comparable fear conditioning paradigm ${ }^{9}$. Accordingly, selective $\mathrm{OX}_{1}$ receptor antagonism has been shown to attenuate anxiety-related behavior in the open field and social interaction test and to reduce neural responses in the central nucleus of the amygdala, bed nucleus of the stria terminalis, periaqueductal gray and in the rostroventrolateral medulla ${ }^{10}$. Correspondingly, pre-treatment with an $\mathrm{OX}_{1}$ receptor antagonist attenuated sodium lactateinduced anxiety-related behavior, locomotor, and cardioexcitatory responses and even worked in a panic-prone strain of rats in a comparable fashion to alprazolam ${ }^{11}$. In a similar vein, pre-treatment with an $\mathrm{OX}_{1}$ receptor antagonist attenuated hypercapnia-induced panic-related behavior and hypertension ${ }^{12}$. Furthermore, systemic $\mathrm{OX}_{1}$ receptor blockage and site-specific blockage in the amygdala have been demonstrated to support the extinction of aversive memories, facilitating the consolidation of cue- and context-dependent fear extinction, potentially via increased infralimbic medial-prefrontal cortex activity ${ }^{13}$. Translating preclinical research to potential clinical applications, human subjects reporting panic-associated symptoms have been found to display elevated qualitative levels of orexin in the cerebrospinal fluid (CSF) compared to subjects without panic-like anxiety ${ }^{11}$. Additionally, chronic treatment with sertraline, a first-line anti-panic drug, has been observed to reduce orexin levels in the CSF of depressed patients ${ }^{14}$.

In sum, given the large body of evidence supporting a key role of particularly the $\mathrm{OX}_{1}$ receptor in hyperarousaland panic-related fear reactions, the gene coding for the $\mathrm{OX}_{1}$ receptor (HCRTR1; chromosome 1p35.2) - containing a potentially functionally relevant $\mathrm{C} / \mathrm{T}$ single nucleotide polymorphism (SNP) (rs2271933) in exon 7 leading to an amino acid exchange from isoleucine to valine (Ile408Val) ${ }^{15-18}$ - is suggested as a prime candidate regarding these phenotypes. Thus, in a multilevel approach, we examined the association between HCRTR1 rs2271933 genotype and panic disorder in two independent samples of patients with panic disorder (PD) with and without agoraphobia (AG) and healthy controls. Within these samples, we explored the possibility of predicting treatment response according to clinical outcome measures based on HCRTR1 rs2271933 genotype in a controlled and randomized exposure-based cognitivebehavioral therapy (CBT) trial. Subsequently, we tested for an association between HCRTR1 rs2271933 genotype and agoraphobic cognitions as measured by the Agoraphobic Cognitions Questionnaire (ACQ) in both PD/AG patients and healthy subjects. Activation in the alerting network as elicited by the attention network task (ANT) comprising the $\mathrm{LC}$ as well as right lateralized fronto-parietal regions particularly important for anxiety-related arousal regulation $^{19,20}$ - was investigated dependent on HCRTR1 rs2271933 genotype and in correlation with dimensional measures of agoraphobic cognitions in an extended sample of healthy probands. Finally, HCRTR1 rs2271933 effects on behavioral avoidance and psychophysiological autonomic arousal during a behavioral avoidance task (BAT) were assessed in $\mathrm{PD} / \mathrm{AG}$ patients. It was hypothesized that HCRTR1 genotype-driven dysfunctional panic-related cognitions and behaviors as well as an oversensitivity of neural network and psychophysiological responses related to arousal and alerting might provide a pathomechanistic framework for an increased vulnerability to PD/AG as well as an impaired response to CBT.

\section{Materials and methods}

For detailed information on samples, genotyping, CBT design, functional magnetic resonance imaging (fMRI) paradigm, BAT, and all statistical analyses refer to the Supplementary Methods.

\section{Samples}

The discovery sample ('MAC sample') for the association study comprised a subsample of the two waves of the 
'Mechanism of Action in CBT' study (MAC) funded by the German Federal Ministry of Education and Research $(\mathrm{BMBF})^{21}$. It consisted of 483 participants of Caucasian origin with available blood samples and primary PD/AG (age $=35.1 \pm 10.7$ years, 341 females [70.6\%], 24 cases without comorbid agoraphobia [5.0\%], 127 cases with comorbid depression [26.3\%], all cases free of psychotropic medication; for additional demographic information including $\mathrm{PD} / \mathrm{AG}$ age of onset and therapeutic history see Supplementary Table 1). For categorical associations, an optimal 1:3 matching with healthy controls (HC) was performed ("optimal matching" as implemented in the $\mathrm{R}$ package MatchIt aims to minimize the global distance measure by matching samples with the smallest average absolute distance across all matched pairs, minimizing the distance between each pair $^{22,23}$ ) based on age and sex against an available dataset of screened healthy controls recruited from the SFBTRR-58 subproject Z02 recruitment waves 1 and $2(n=2382$; age $=25.2 \pm 5.8$ years, 1486 females [62.4\%]). Matching was limited to a $1: 3$ ratio due to the availability of healthy female control samples (see below) ${ }^{24}$. In addition, the total sample of MAC PD/AG patients and SFBTRR-58 Z02 healthy controls had been characterized for scores on the German version of the Agoraphobic Cognitions Questionnaire $(\mathrm{ACQ})^{25}$.

The replication sample ('Münster sample') consisted of 130 patients with primary PD/AG (age $=35.7 \pm 11.1$ years, 84 females [64.6\%], 40 cases without comorbid agoraphobia [30.8\%], 46 cases with comorbid depression [35.4\%]) and 130 healthy controls (age $=35.8 \pm 10.1$ years) recruited at the Department of Psychiatry and Psychotherapy, University of Münster, Germany, supplemented with healthy controls recruited from the SFBTRR58 subproject Z02 recruitment waves 1 and 2 and not used in the matching process for the discovery sample, in order to again reach a PD/AG:HC ratio of 1:3. Study inclusion criteria were comparable between the discovery and replication samples and based on DSM-IV criteria (see Supplementary Methods).

Patients and healthy controls gave full written informed consent; the studies were in agreement with the Declaration of Helsinki and approved by the respective local ethics committees.

\section{Cognitive-behavioral psychotherapy (CBT)}

Within the MAC study, patients underwent CBT sessions following a manualized structure and administered twice weekly, with a total of 12 sessions $^{26}$. A subsample of patients was available for genetic analyses $(n=189$; age $=$ $35.4 \pm 11.0$ years, 139 females [73.5\%]).

Primary outcome measures investigated in the MAC study were the Hamilton Anxiety Rating Scale (HAM-A), the Clinical Global Impressions Scale (CGI), the Panic and
Agoraphobia Scale (PAS), the number of panic attacks in the week prior to assessment and the Mobility Inventory for Agoraphobia Avoidance "Alone" Scale (MI), all of which were assessed at baseline and post $\mathrm{CBT}^{26}$.

\section{Functional MRI}

A total of 94 healthy subjects of Caucasian descent performed the fMRI task (age $=28.5 \pm 8.9$ years, 63 females [67.0\%], 44 samples drawn from the SFBTRR-58 subproject $Z 02$ recruitment waves 1 and 2 and 50 samples additionally recruited at the Department of Psychiatry, Psychosomatics, and Psychotherapy, University of Würzburg, Germany). Analyses focused on neural activity of the alerting network as evaluated via the Attentional Network Task (ANT) capturing alerting, orienting and executive functions. The paradigm and data acquisition have been published previously (see Supplementary Methods $)^{19}$.

\section{Behavioral avoidance task (BAT)}

A subsample of the 'MAC sample' during recruitment wave 1 underwent the behavioral avoidance task ${ }^{27,28}$. The protocol for the BAT comprised of an exposure to a dark, small and closed test chamber which patients would first sit in front of with the door open for $10 \mathrm{~min}$ ('anticipation phase') before being locked in for a maximum of $10 \mathrm{~min}$ ('exposure phase'), followed by the 'recovery phase', again, in front of the open door for $8 \mathrm{~min}$. Avoidance behavior and psychophysiological measures were ascertained in all phases (see Supplementary Methods; for additional pretreatment heart rate and pre- and post-treatment subjective fear readouts refer to the Supplementary Results and Discussion). Patients were explicitly allowed to terminate the BAT protocol at any time. Dependent on their behavior during the task, patients were categorized into one of three groups: 'passive avoidance' (no attempt of exposure in the BAT chamber), 'active avoidance' (flight during the $10 \mathrm{~min}$ period of exposure) and 'no avoidance' (no attempt of escape during the exposure). After completion of each phase, patients were instructed to rate their subjective experience of fear on a visual analogue scale of 1 to 10 . Mean heart rates during the three phases were recorded via a continuous electrocardiogram.

Full HCRTR1 rs2271933 genotype and BAT data was available for $271 \mathrm{PD} / \mathrm{AG}$ patients pre-CBT (age $=36.1 \pm$ 10.9 years, 202 females [74.5\%]) and for 183 patients post$\mathrm{CBT}$, excluding those randomized to a wait-list-control group $(n=49)$, treatment drop-outs $(n=33)$ and those who failed to repeat the BAT after therapy $(n=6)$.

\section{Genotyping}

All samples were genotyped for the HCRTR1 rs2271933 using a PCR-restriction-fragment-length-polymorphism (RFLP) assay (see Supplementary Methods). 


\section{Results}

Categorical diagnosis of panic disorder with and without comorbid agoraphobia

HCRTR1 rs2271933 allele and genotype frequencies and full test statistics, $p$-values, odds ratios (OR) and 95\% confidence intervals (CI) for the discovery sample (MAC study) and the replication sample (Münster sample) are given in Table 1.

In the MAC discovery sample, rs2271933 $\mathrm{T}$ allele loading was significantly associated with PD/AG in the Cochran-Armitage test and the allelic Pearson's $\chi^{2}$-test. Furthermore, the genotype model Pearson's $\chi^{2}$-test supported a recessive effect of the $\mathrm{T}$ allele in PD/AG (TT vs $\mathrm{CC} / \mathrm{CT}$ ). Stratification by sex revealed a predominantly female association effect.

The association between the rs2271933 T allele and PD/ AG was successfully validated in the Münster replication sample via the Cochran-Armitage test and Pearson's $\chi^{2}$ tests for the allelic and recessive genotype models. Again, the genetic association was predominately driven by the female subsample of the Münster sample.

Meta-analytical combination of the total discovery and replication samples (613 PD/AG patients, 1839 controls) in a fixed-effects model resulted in a significantly increased OR for both, the recessive (OR $1.82[95 \% \mathrm{CI}=1.83-2.41]$, $\mathrm{Z}=4.18, p=2.9 \times 10^{-5}$ ) and the allelic model (OR 1.51 [95\%CI = 1.29-1.77], $\mathrm{Z}=5.06, p=4.2 \times 10^{-7}$ ) (Fig. 1). Further meta-analyses confirmed the increased PD/AG OR in females only for the recessive (OR $2.59[95 \% \mathrm{CI}=$ 1.81-3.69], $\mathrm{Z}=5.25, p=2.0 \times 10^{-4}$ ) and allelic model (OR $1.78[95 \% \mathrm{CI}=1.46-2.17], \mathrm{Z}=5.73, p=9.8 \times 10^{-9}$ ).

\section{CBT treatment response in panic disorder with and without comorbid agoraphobia}

In the MAC PD/AG patient subsample undergoing CBT (HCRTR1 rs2271933 genotype distribution: $\mathrm{CC}=58, \mathrm{CT}$ $=78$, TT $=53$ ), TT genotype carriers displayed a significantly decreased treatment response as assessed via HAM-A scores (TT vs CC and TT vs CT), CGI scores (TT vs CC; trend towards significance for TT vs CT) and PAS scores (TT vs CC; trend towards significance for TT vs $\mathrm{CT})$. Furthermore, in $\mathrm{T}$ allele homozygotes a trend towards a significantly diminished treatment response on the MI was discerned when compared to CT heterozygotes. Applying a recessive model (TT vs $\mathrm{CC} / \mathrm{CT}$ ) the reduced treatment response effects in $\mathrm{T}$ allele homozygotes remained significant for the HAM-A $(p=1.5 \times$ $\left.10^{-3}\right)$, CGI $(p=0.031)$ and PAS $(p=0.044)$ scores. For full test statistics, $p$-values, ORs and 95\% CIs refer to Table 2.

\section{Dimensional anxiety traits in panic disorder with and without comorbid agoraphobia and in healthy controls \\ Within the total MAC PD/AG patient sample, $\mathrm{T}$ allele loading was significantly associated with increased ACQ}

scores (by 1.90 points per $\mathrm{T}$ allele, 95\%CI: $0.76-3.04$ points; allelic model, $p=0.027)$. When evaluating the effect of the rs2271933 T allele on ACQ scores within the sample of 2382 healthy subjects, $\mathrm{T}$ allele loading was again significantly associated with an increased ACQ score (by 0.50 points per $\mathrm{T}$ allele, $95 \% \mathrm{CI} 0.35-0.65$ points; allelic model, $p=0.014$ ).

\section{Alerting network in healthy controls}

In the sample of healthy probands performing the fMRI Attention Network Task (HCRTR1 rs2271933 genotype distribution: $\mathrm{CC}=23, \mathrm{CT}=50$, $\mathrm{TT}=21$ ), two significant clusters of neural activation in the alerting condition were detected when applying a gene dosage analysis (Fig. 2a): One cluster within the right inferior frontal gyrus (IFG, $x$ $=34, y=44, z=-10$ ) with decreasing activation associated with increased $\mathrm{T}$ allele counts $(Z=3.1, p=0.041$, FDR-corrected at the voxel level) and one cluster within the bilateral locus coeruleus (LC, $x=-14, y=-16, z=$ $-2)$ with increasing activation associated with increased T allele counts $(Z=3.2, p=0.031$, FDR-corrected at the voxel level).

In order to address the relation between neural activation of the alerting system, rs2271933 $\mathrm{T}$ allele count and dimensional anxiety traits (ACQ), interaction analyses were performed. Neural activation in the LC cluster significantly correlated with increased ACQ scores in TT genotype carriers and decreased ACQ scores in CC genotype carriers (Fig. 2b) (TT >CC/CT: $Z=3.5, p=0.032$; $\mathrm{TT}>\mathrm{CC}: Z=3.6, p=0.028$; TT $>\mathrm{CT}: Z=1.5, p=0.210)$. A negative correlation between ACQ scores and right IFG activation in TT genotype carriers reached trend-wise significance (TT < CC/CT: $Z=2.1, p=0.084$; TT < CC: $Z$ $=1.9, p=0.089$; TT $<$ CT: $Z=0.01, p=0.981)$.

There was no genotype effect on accuracy and reaction time on the behavioral level. To assess the relation between ACQ scores and behavioral parameters, partial correlations were performed using sex as nuisance variable: ACQ scores did not significantly influence behavioral performance $\left(R_{\text {accuracy }}=0.039, p=0.714 ; R_{\text {reaction }}\right.$ time $\left.=-0.055, p=0.600, R_{\text {alerting }}=0.075, p=0.480\right)$.

\section{Behavioral avoidance task (BAT) in panic disorder with and without comorbid agoraphobia}

Pre-treatment assessment (HCRTR1 rs2271933 genotype distribution: $\mathrm{CC}=79, \mathrm{CT}=120, \mathrm{TT}=72$ ): During the BAT, frequency of avoidance behavior was significantly more pronounced along with an increasing number of rs2271933 T alleles (categorical BAT analysis; passive avoidance by genotype: $\mathrm{CC}=2$ [2.5\%], $\mathrm{CT}=15$ [12.5\%], TT $=12$ [16.7\%]; active avoidance by genotype: $\mathrm{CC}=19$ [24.1\%], CT $=21$ [17.5\%], TT $=15$ [20.8\%]; no avoidance by genotype: $\mathrm{CC}=58 \quad[73.4 \%], \quad \mathrm{CT}=84$ [70.0\%], TT $=45$ [62.5\%]; linear-to-linear trend: $\chi^{2}(1)=$ 


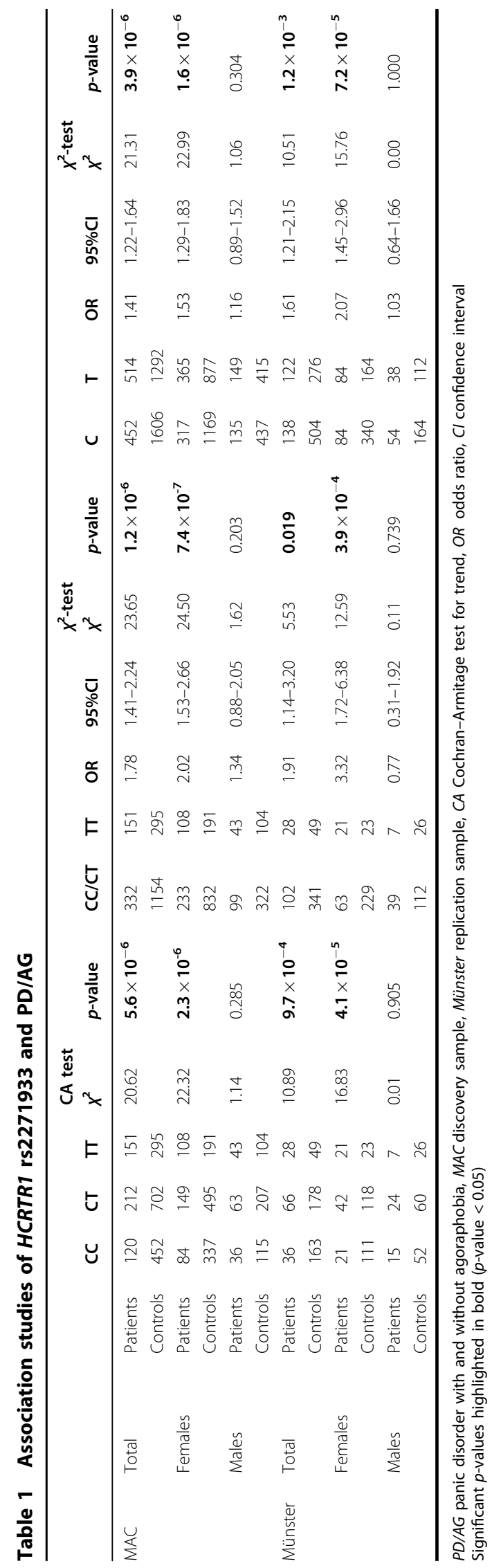

5.172, $p=0.023)$. Concordantly, mean duration of tolerated BAT exposure trended towards a significant decrease with increasing $\mathrm{T}$ allele loading (dimensional BAT analysis; linear trend $p=0.073$; Fig. 3a).

In those patients entering the test chamber, the mean heart rate during BAT anticipation did not significantly differ between patients showing active avoidance vs no avoidance during BAT exposure (Behavior $\mathrm{F}(1,186)=$ $0.338, p=0.562$ ). Also, no genotype effect was observed during the anticipation phase (Genotype $\mathrm{F}(2,186)=1.718$, $p=0.182$; Genotype $\times$ Behavior $\mathrm{F}(2,186)=2.476, \quad p=$ 0.087). However, while the initial heart rate increase during BAT exposure did not differ between genotype groups in active avoiders $(\mathrm{F}(2,42)=0.615, p=0.546)$, the heart rate response decreased with increasing number of $\mathrm{T}$ alleles in non-avoiding patients (linear trend: $p=$ 7.7.10 ${ }^{-3}$; Fig. 3b).

Post-treatment assessment (HCRTR1 rs2271933 genotype distribution: $\mathrm{CC}=57, \mathrm{CT}=75, \mathrm{TT}=51$ ): In patients showing active or passive avoidance behavior during the BAT assessment prior to therapy, the increase of the mean duration of tolerated BAT exposure from pre- to post-assessment diminished with an increasing number of rs2271933 T alleles (linear trend $p=0.043$; Fig. 3c).

Accordingly, the pre- to post-assessment reduction of the initial heart rate response from anticipation to exposure phase attenuated with an increasing number of rs2271933 $\mathrm{T}$ alleles (linear trend $p=6.0 \times 10^{-3}$; Fig. 3d) irrespective of whether patients showed active avoidance or not during the BAT pre-treatment assessment (Genotype $\times$ Behavior $\mathrm{F}(2,119)=0.294, p=0.746)$.

\section{Discussion}

Our data demonstrate an association between the HCRTR1 rs2271933 T allele and PD/AG in a Caucasian population, including a successful replication and metaanalysis. The HCRTR1 rs2271933 driven nonsynonymous Ile408Val substitution resides in the receptor's C-terminus and is therefore likely to be part of a domain involved in protein-protein interaction, but as of yet, it remains to be experimentally tested, whether the SNP affects expression levels, effector coupling or dimerization of the $\mathrm{OX}_{1}$ receptor ${ }^{29}$. Based on the above mentioned findings the $\mathrm{T}$ allele - presently discerned to mediate hyperarousal- and/or panic-related anxiety behavior - is hypothesized to enhance $\mathrm{OX}_{1}$ receptor signaling (e.g., on the expression level, or due to reduced cycling/desensitization) and thereby to increase the orexinergic tone in key brain areas of the arousal/alerting system. Interestingly, the genetic association was mainly driven by the female subsamples of the present samples, a phenomenon already observed in other neuropeptideand monoamine/catecholamine-related polymorphisms implicated in PD/AG pathology ${ }^{30,31}$. Indeed, sexually 
a Recessive Model, Total Sample (TT vs CC/CT)

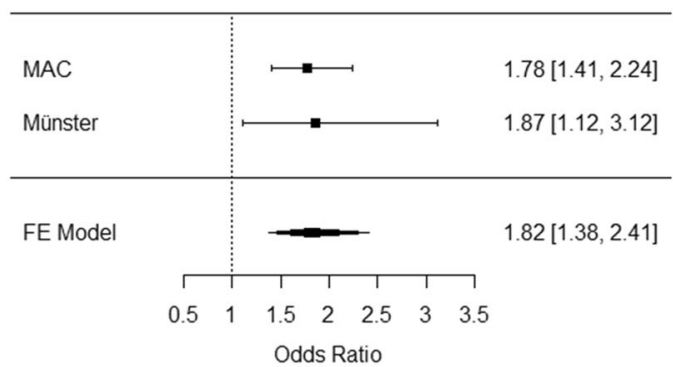

b Recessive Model, Female Sample (TT vs CC/CT)

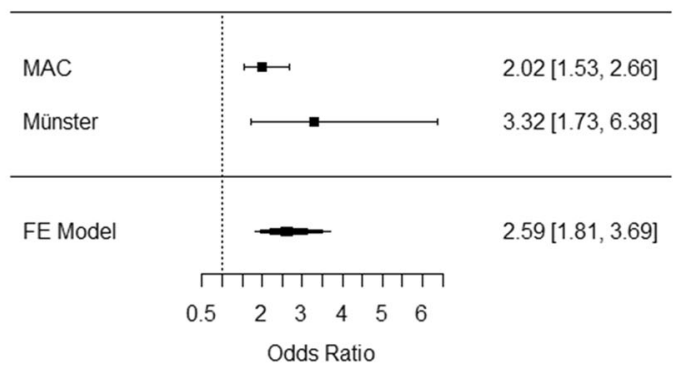

C

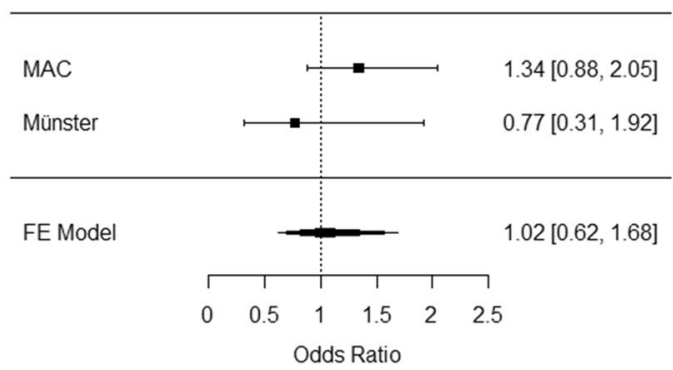

Allelic Model, Total Sample (T vs C)

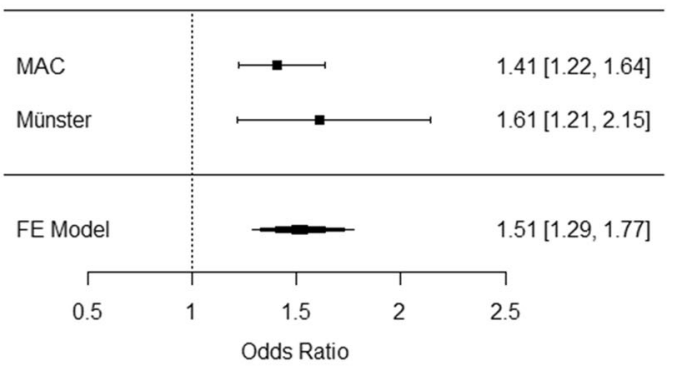

Allelic Model, Female Sample (T vs C)

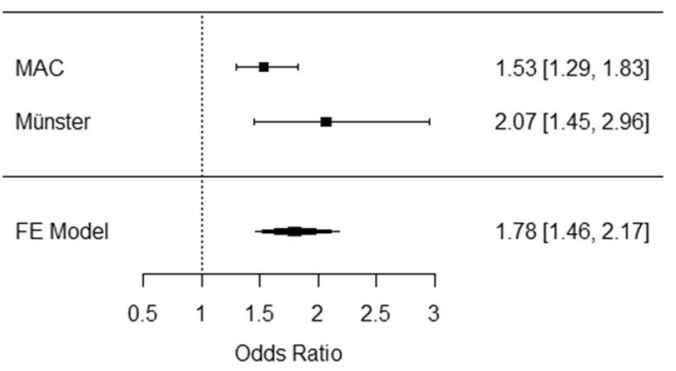

Allelic Model, Male Sample (T vs C)

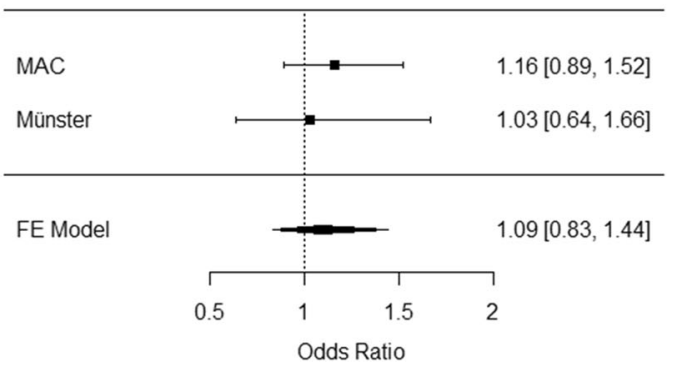

Fig. 1 Forest plots of HCRTR1 rs2271933 meta-analyses of association with PD/AG. PD/AG = panic disorder with and without agoraphobia; MAC = discovery sample; Münster = replication sample. FE = fixed-effects. Values in brackets represent $95 \%$ confidence intervals of odds ratios

dimorphic expression of orexin and the orexin receptors in the CNS has been described in mice ${ }^{32}$. Furthermore, it has been shown that restraint stress-induced HPA-axis activation and cognitive impairments via increased glucocorticoid receptor-dependent orexin expression occur exclusively in female rats and can be prevented by chemogenetic inhibition of hypothalamic orexinergic neurons, pointing to a sex-specific role of orexins and their receptors in stress susceptibility ${ }^{33}$. The only other study evaluating orexin receptor polymorphisms in $\mathrm{PD} / \mathrm{AG}$ failed to show genetic association for HCRTR1 rs2271933, but rather reported a significant finding for the HCRTR2 rs2653349 A allele, again predominantly in the female subsample $^{15}$. It should be noted, however, that in this previous study PD/AG sample size was limited, controls were not screened for $\mathrm{PD} / \mathrm{AG}$ and no independent replication was provided.
Moreover, the HCRTR1 rs2271933 T allele was found to confer significantly poorer outcomes on clinical assessments (HAM-A, CGI) and a self-report measurement (PAS) following exposure-based CBT. Given that changes in vigilance for threat information have been demonstrated to precede and predict clinical changes in exposure-based $\mathrm{CBT}$ for $\mathrm{PD} / \mathrm{AG}^{34}$, an altered orexinergic tonus differentially driven by rs2271933 genotype and affecting arousal/alerting processes might influence CBT outcome in PD/AG. The present finding extends the emerging body of evidence for biomarkers of psychotherapy response prediction in PD/AG, so far mainly focusing on genetic variations related to serotonergic and monoaminergic neurotransmission such as the monoamine oxidase A $(M A O A)$ upstream variable number of tandem repeats (uVNTR) $\mathrm{SNP}^{35}$, to the arousal-related orexin system. 


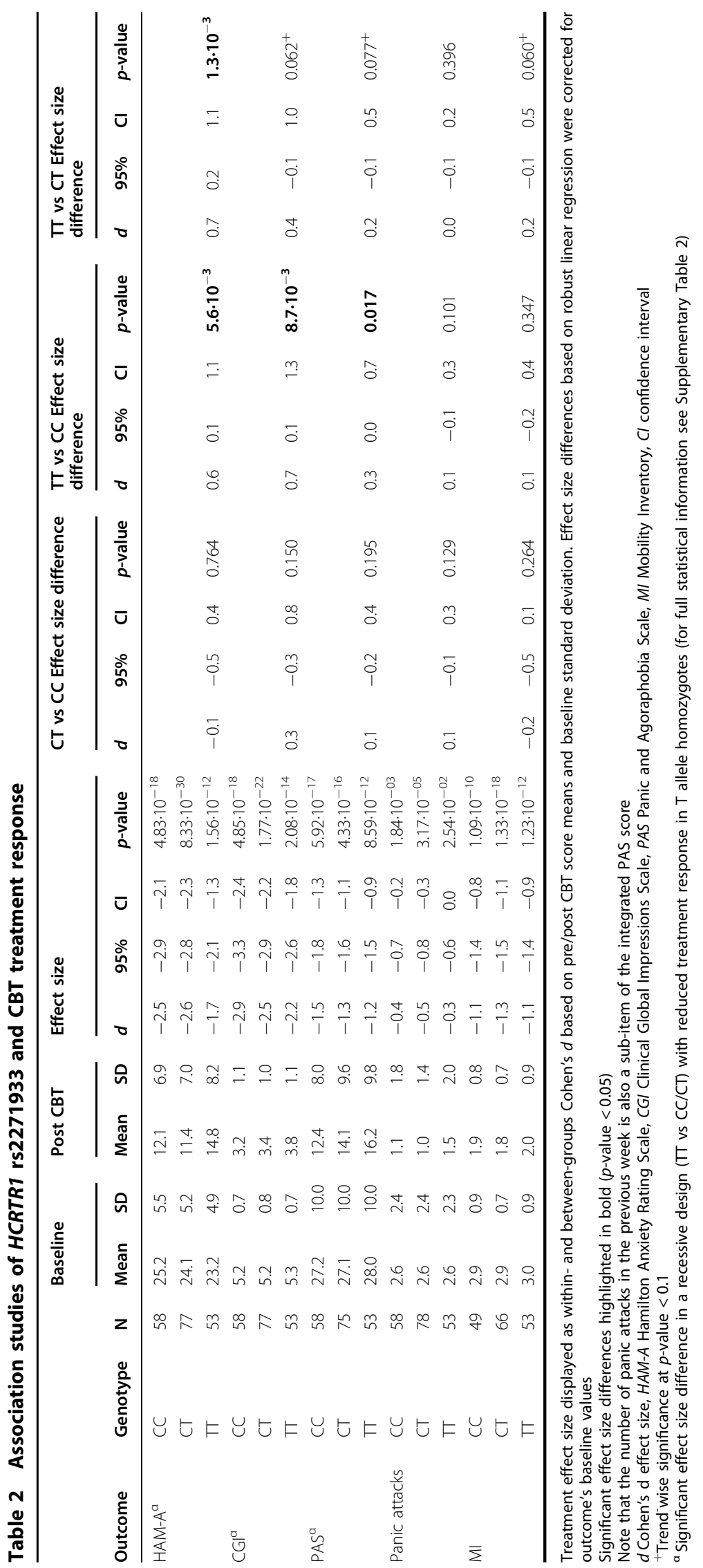




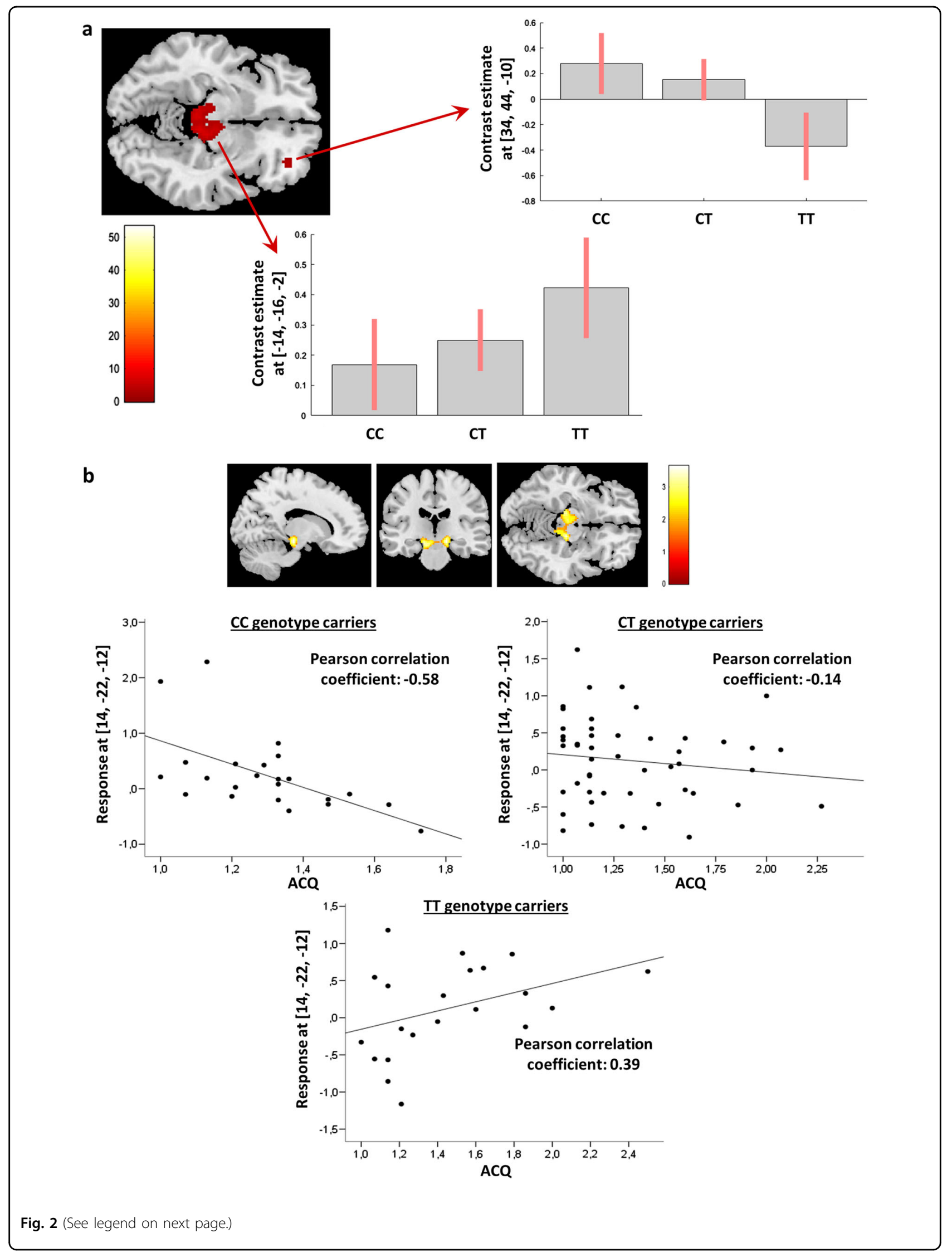


Fig. 2 HCRTR1 rs2271933 genotypes and the alerting system. a Left: Significant HCRTR1 rs2271933 gene-dose effect clusters in the alerting system are overlaid on a single subject T1 anatomical image. Right and bottom: Bar plots represent the HCRTR1 rs2271933 genotype-dose effect in terms of contrast estimates at the local maxima. $\mathbf{b}$ Effects of Agoraphobic Cognitions Questionnaire (ACQ) scores on the alerting network. Top: Activation clusters rendered on a single subject brain surface. Bottom: Correlations of ACQ scores with locus coeruleus (LC) activation. In HCRTR1 rs2271933 T allele homozygotes, ACQ scores correlated positively with LC neural activity, while there was a negative correlation in C allele homozygotes (see Results)

In the attempt to explore intermediate phenotypic levels potentially contributing to the observed genotype effects, the HCRTR1 rs2271933 $\mathrm{T}$ allele count was found to be associated with increased agoraphobic cognitions (ACQ) scores within the MAC PD/AG patient sample and in a large sample of psychiatrically healthy subjects. It has already been shown that increased ACQ scores correlate directly with increased bodily sensations in feared situations in anxiety disorder patients and in psychiatrically healthy controls ${ }^{36}$. Accordingly, elevated ACQ scores prior to a $\mathrm{CO}_{2}$ challenge predicted the intensity of frightening cognitions after inhalation, both in a PD/AG and a healthy control sample ${ }^{37}$, thus providing a cognitive link between arousal-related interoceptive sensations and PD/AG risk. Also, the presently observed therapygenetic effect might in part be explained by the fact that the HCRTR1 rs2271933 $\mathrm{T}$ allele predicted increased ACQ scores, given that catastrophic misinterpretations during a 12-week CBT program have previously been shown to impair therapeutic success ${ }^{38}$. In sum, the HCRTR1 rs2271933 $\mathrm{T}$ allele might confer an increased proneness to PD/AG-related catastrophizing thought patterns and thereby increased PD/AG risk as well as treatment failure.

On a neural level, we observed a significant effect of HCRTR1 rs2271933 genotype on alerting network activation in healthy subjects, with an increased $\mathrm{T}$ allele count associated with decreased neural response in the right inferior frontal gyrus (IFG) and an increased response in the locus coeruleus (LC). Remarkably, LC activation varied positively in function of the ACQ in T allele homozygotes, signifying that a higher ACQ score was linked to a stronger neural bottom-up processing of internal stimuli. At the same time, reduced activation in the IFG, a region which has been hypothesized to represent the modulation of warning signals on the level of alertness, might indicate an impaired top-down inhibition of the subcortical alerting network ${ }^{39}$. Orexin has been reported to activate noradrenergic LC neurons and thereby heightens arousa ${ }^{40}$. Notably, in terms of fear conditioning Hcrtr1 knockout mice displayed impaired freezing responses and reduced neural activation of the lateral amygdala, which could be rescued by adeno-associated viral-mediated restoration of Hcrtr1 expression in the $\mathrm{LC}^{8}$. Local microinjections of an $\mathrm{OX}_{1}$ receptor antagonist and subsequent optogenetic specific stimulation of orexin fibers in the LC further validated the importance of $\mathrm{OX}_{1}$ receptor-controlled norepinephrinergic $\mathrm{LC}$ neurons in the formation of fear memories 9 . Additionally, the information that hypercapnia leads to increased brainstem activation in PD/AG patients compared to healthy controls ${ }^{41}$, and the positive correlation between LC activation and ACQ scores in TT genotype carriers, advocate for an increased risk of developing PD/AG due to a combination of elevated attention to autonomic bodily functions and increased somatic arousal due to dysfunctional cortical attentional networks ${ }^{20,42}$. Moreover, overactivation of the LC-amygdala circuit has been linked to increased fear generalization, which was reversible under $\mathrm{OX}_{1}$ receptor antagonist treatment ${ }^{43}$. Since individuals with higher trait anxiety and amygdala hyperresponsivity have been proven to exhibit an increased resistance to fear extinction ${ }^{44}$, overactivation of the LC in alerting conditions presently identified in HCRTR1 rs2271933 T allele homozygotes, may have significantly impaired CBT efficiency of exposure-based extinction learning, as observed in the treatment response analysis. In sum, the present results propose a HCRTR1 genotype by phenotype interaction in terms of an imbalance between frontal top-down and brain stem bottom-up control in the alerting network, reflecting a putative neuronal model of hyperarousal in the perception and processing of fear-related bodily symptoms with relevance to PD/AG pathology as well as therapy resistance due to abnormal stimulus generalization and extinction.

Finally, the HCRTR1 rs2271933 $\mathrm{T}$ allele was associated with increased rates of avoidance behavior and a decreased capacity to endure psychophysiological activation in PD/AG patients during a fear-provoking behavioral avoidance test (BAT). Surprisingly, $\mathrm{T}$ allele loading was associated with decreased physiological fear reactivity to BAT exposure as indicated by the measured heart rate responses, however, only in BAT non-avoiding but not in BAT escaping patients. In $\mathrm{T}$ allele homozygotes displaying no active avoidance, no substantial fear increase from anticipation to exposure was observed. Thus, if the patients' arousal system is activated, $\mathrm{T}$ allele carriers might be significantly more likely to avoid the fearinducing situation, manifesting in escape from the phobic environment, resulting in more frequent avoidance behavior and, in turn, lower levels of autonomic fear in 

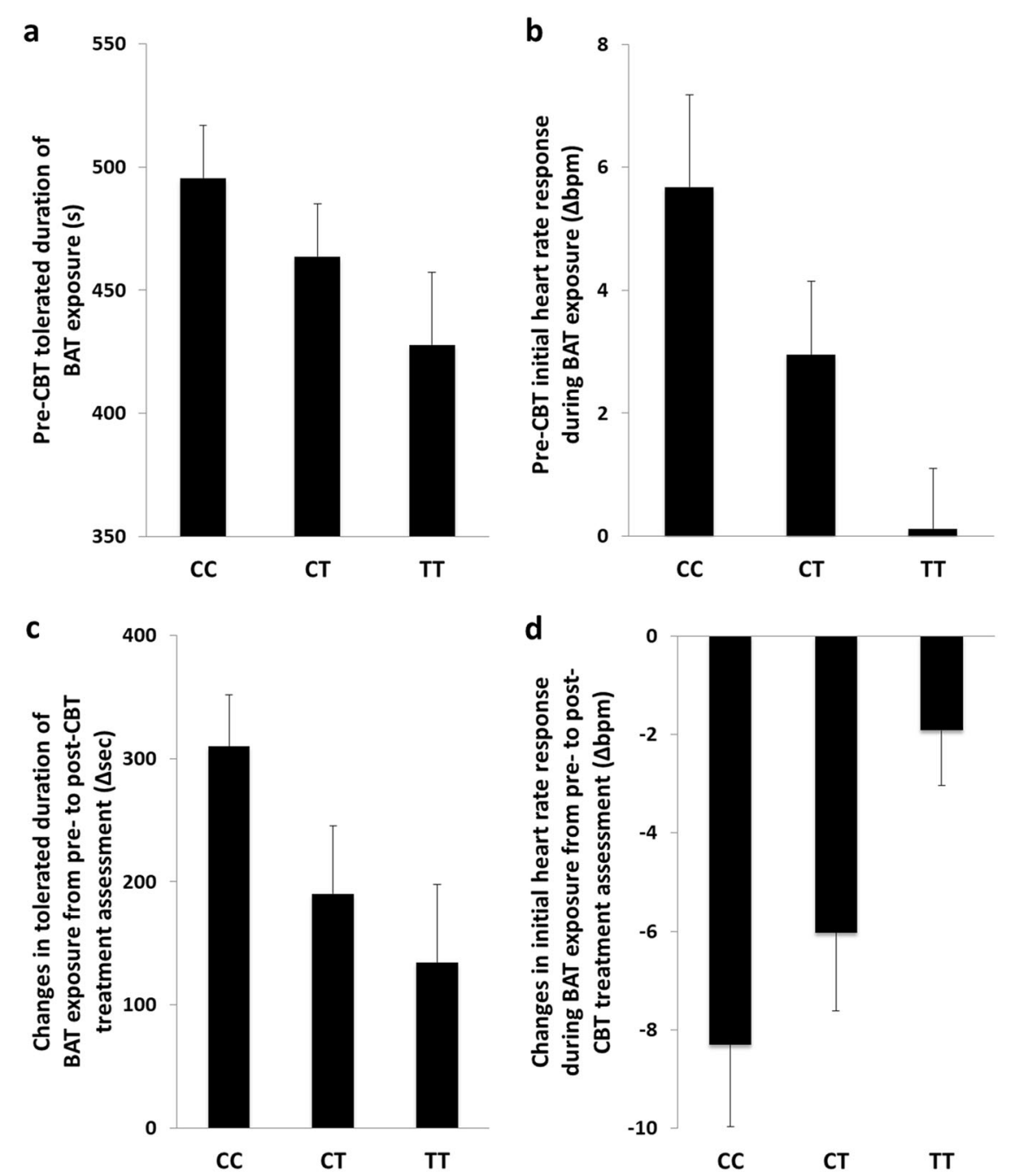

Fig. 3 HCRTR1 rs2271933 T allele loading and behavioral avoidance task (BAT) outcomes. a T allele loading vs pre-treatment (pre-CBT) duration of exposure (s). $\mathbf{b}$ T allele loading vs pre-treatment initial heart rate response $(\Delta \mathrm{bpm})$ during exposure, i.e., increase from last minute of anticipation to first minute of exposure, in non-avoiding patients. $\mathbf{c}$ T allele loading vs the pre- to post-treatment difference in tolerated duration of exposure $(\Delta s)$. d T allele loading vs the pre- to post-treatment difference of the initial heart rate response $(\Delta \mathrm{bpm})$ during exposure. All values are presented as means \pm standard error (SE)

non-avoiding subjects as compared to non-risk-allele carriers. Our results are in line with current learning theories in $\mathrm{PD} / \mathrm{AG}$, stating that chances of experiencing another panic attack are heightened once they have been associated with internal cues of elevated arousal. This could explain why $\mathrm{T}$ allele carriers would most likely choose to avoid phobic exposure in general and thus are more prone to develop PD/AG from an initial panic $\operatorname{attack}^{45}$. Additionally, we found an increased maintenance of avoidance behavior during the BAT after CBT treatment with an increasing number of rs2271933 $\mathrm{T}$ alleles that went along with a lack of a reduction of physiological fear reactivity from pre- to post-treatment assessment. Reduced tolerance of fear activation during phobic exposure - as displayed by $\mathrm{T}$ allele carriers could possibly be detrimental for safety learning and thus impede successful fear extinction, as observed in the treatment response analysis ${ }^{46}$. In line with this hypothesis, agoraphobic avoidance was the most consistent predictor of decreased improvement in CBT for PD/AG in a recent systematic literature review ${ }^{47}$. Taken together, the present data support the notion, that the HCRTR1 rs2271933 T allele might confer a genetic risk of decreased resilience to phobic exposure in $\mathrm{PD} / \mathrm{AG}$ resulting in pronounced avoidance behavior.

The present results should be considered in the light of some limitations. Besides the necessity to replicate our findings in even larger samples, particularly when 
stratifying for sex, several factors not accounted for and thus potentially confounding the present results such as possible moderation effects by childhood trauma and recent life events should be controlled for in future studies. Given that the healthy controls were younger than the PD/AG patient populations, despite optimal matching of the available samples, we cannot exclude a potential bias in the results given the possibility that they might develop a PD/AG later in life. Along these lines, epigenetic mechanisms like DNA methylation mediating environmental influences and governing gene function remain to be addressed ${ }^{48}$. The decision to investigate only one SNP in the HCRTR1 gene was based on its likely functional relevance and due to it having been investigated in the majority of association studies on HCRTR1 gene variation in neuropsychiatric phenotypes ${ }^{15-18}$. However, in order to capture the entire HCRTR1 gene information and to exclude potential effects of population stratification, a tagging SNP approach would have to be applied. Additionally, it should be noted that SNPs in linkage disequilibrium with rs2271933 could causally drive the reported findings, yet this would optimally require whole genome sequencing data to be ruled out entirely. Future studies are warranted to experimentally delineate the presently only assumed functionality of HCRTR1 rs2271933 and its interaction with other established psychiatric candidate gene variants. Particularly, the interaction of HCRTR1 and HCRTR2 gene variation remains to be elucidated given preclinical evidence for a bi-directional control of anxiety via orexin receptors ${ }^{49}$.

In conclusion, we have gathered converging evidence for a both patho-genetic and therapy-genetic role of the HCRTR1 rs2271933 T allele in PD/AG. In synopsis with translational preclinical studies, the present findings suggest a potential value of $\mathrm{OX}_{1}$ receptor antagonists as a novel treatment strategy in arousal- and panic-related anxiety $^{10-13}$. Also, applying a personalized treatment approach, HCRTR1 gene variation, along with physiological, neurocircuit, behavioral and self-report measures, might aid in predicting treatment response and allow for tailoring therapeutic interventions to the individual patient's risk factor constellation ${ }^{50,51}$.

\footnotetext{
Acknowledgements

The study was funded by the Deutsche Forschungsgemeinschaft (DFG, German Research Foundation) - project number 44541416-TRR 58 (projects Z02 to K.D., J.D., A.R., M.R., and P.P., and C02 to K.D. and S.N.). The MAC study was funded by the German Federal Ministry of Education and Research (BMBF; project no. 01GV0615) as part of the BMBF Psychotherapy Research Funding Initiative. The principal investigators (PIs) of the centers with respective areas of responsibility in the MAC study are: V. Arolt (Münster: Overall MAC Program Coordination), H.U. Wittchen (Dresden: PI for the Randomized Clinical Trial and Manual Development), A. Hamm (Greifswald: PI for Psychophysiology), A.L. Gerlach (Münster: PI for Psychophysiology and Panic subtypes), A. Ströhle (Berlin: PI for Experimental Pharmacology), T. Kircher (Marburg: Pl for functional neuroimaging) and J. Deckert (Würzburg: PI for Genetics). Additional site directors in the randomized controlled trial component of the program are as
}

follows: G.W. Alpers (Würzburg), T. Fydrich and L. Fehm (Berlin-Adlershof) and T. Lang (Bremen). Acknowledgements and staff members by site-Greifswald (coordinating site for Psychophysiology): Christiane Pané-Farré, Jan Richter, Susan Richter, Matthias von Rad; Berlin-Charité (coordinating Center for Experimental Pharmacology): Harald Bruhn, Anja Siegmund, Meline Stoy, André Wittmann; Berlin-Adlershof: Irene Schulz; Münster (Overall MAC Program Coordination, Genetics and Functional Neuroimaging): Andreas Behnken, Katharina Domschke, Adrianna Ewert, Carsten Konrad, Bettina Pfleiderer, Christina Uhlmann, Peter Zwanzger; Münster (coordinating site for psychophysiology and subtyping): Judith Eidecker, Swantje Koller, Fred Rist, Anna Vossbeck-Elsebusch; Marburg/Aachen (coordinating center for functional neuroimaging): Barbara Drüke, Sonja Eskens, Thomas Forkmann, Siegfried Gauggel, Susan Gruber, Andreas Jansen, Thilo Kellermann, Isabelle Reinhardt, Nina Vercamer-Fabri; Dresden (coordinating site for data collection, analysis, and the RCT): Franziska Einsle, Christine Froehlich, Andrew T. Gloster, Christina Hauke, Simone Heinze, Michael Hoefler, Ulrike Lueken, Peter Neudeck, Stephanie Preiß, Dorte Westphal; Würzburg Psychiatry Department (coordinating center for genetics): Andreas Reif, Caro Gagel; Würzburg Psychology Department: Julia Duerner, Hedwig Eisenbarth, Antje B. M. Gerdes, Harald Krebs, Paul Pauli, Silvia Schad, Nina Steinhäuser; Bremen: Veronika Bamann, Sylvia Helbig-Lang, Anne Kordt, Pia Ley, Franz Petermann, Eva-Maria Schroeder. Additional support was provided by the coordinating center for clinical studies in Dresden (KKS Dresden): Xina Graehlert and Marko Käppler. This work is also part of the German multicenter trial: Mechanisms of CBT treatment effects in patients with panic disorder and panic disorder with agoraphobia: The role of interoceptive exposure and fear augmentation (MCBT-PDAS). The study is funded by the German Federal Ministry of Education and Research (BMBF, 01GV0614) as part of the larger BMBF Psychotherapy Research Funding Initiative Improving the Treatment of Panic Disorder. Principal investigators (PI) with respective areas of concentration of the MCBT-PDAS are Alfons Hamm (Greifswald: PI Psychophysiology); Thomas Lang (Bremen: Study Director for the Randomized Clinical Trial (RCT) and Manual Development); Alexander L. Gerlach (Münster: PI Panic subtypes); Georg W. Alpers (Mannheim: PI Ambulatory assessment); Christiane Pané-Farré (Greifswald: PI Psychophysiology and Panic Disorder); Tilo Kircher (Marburg: PI for functional neuroimaging), and Jürgen Deckert (Würzburg: PI for Genetics). Additional site directors in the RCT component of the program are Winfried Rief (Marburg), and Paul Pauli (Würzburg). Centers of the Research Network: Volker Arolt (Münster: Overall Network Coordination), Hans-Ulrich Wittchen (Dresden), Andreas Ströhle (Berlin). Acknowledgements and staff members by site: Bremen (coordinating center for the multicenter trial): Veronika Bamann, Sandra Cammin, Sarah Czilwik, Kira Geisler, Sylvia Helbig-Lang, Kirsten Helmes, Anne Kordt, Tanja Leonhard, Mila Plett-Perelshteyn, Christian Soltau, Juliane Sülz, Maxie von Auer; Greifswald (coordinating site for psychophysiology): Anett Hoffmann, Jan Richter; Mannheim (coordinating center for ambulatory assessment): Christoph Biwer, Elisabeth Borgmann, Antje Gerdes, Otto Martin, Kristina Steinbach, Bettina Stemmler, Andrew White; Marburg (coordinating center for functional neuroimaging): Tobias Fehlinger, Andreas Jansen, Nikita Jegan, Carsten Konrad, Marion Mickeler, Silke Rusch, Katrin Schlötterer, Benjamin Straube, Mareike Stumpenhorst, Katrin Wambach, Yunbo Yang; Münster (coordinating site for panic subtypes): Susanne Kettler, Anna Vossbeck-Elsebusch; Würzburg Psychiatry Department (coordinating center for genetics): Carola Gagel, Andreas Reif, Heike Weber; Würzburg Psychology Department: Almut Friedl-Huber, Harald Krebs, Caroline Ott, Nina Steinhäuser; Additional support was provided by the coordinating center for clinical studies in Dresden (KKS Dresden): Marko Käppler. The study was registered with the NCT01323556. Further support was obtained from the German Federal Ministry of Education and Research (BMBF; project no. 01EE1402F, PROTECT-AD) to KD, JD and HUW, and the IZKF Würzburg N-262 to K.D., S.N., and G.H. R.K. was supported by the DFG grant KA1623/3-1. We are grateful to all individuals who participated in this study. We gratefully acknowledge the skillful technical support by C. Gagel.

\footnotetext{
Author details

${ }^{1}$ Department of Psychiatry and Psychotherapy, Medical Center - University of Freiburg, Faculty of Medicine, University of Freiburg, Freiburg, Germany. ${ }^{2}$ Department of Psychiatry, Psychosomatics and Psychotherapy, Center of Mental Health, University Hospital of Würzburg, Würzburg, Germany. ${ }^{3}$ Department of Biological and Clinical Psychology/Psychotherapy, University of Greifswald, Greifswald, Germany. ${ }^{4}$ Epilepsy Center, Medical Center University of Freiburg, Faculty of Medicine, University of Freiburg, Freiburg,
} 
Germany. ${ }^{5}$ Department of Physiology, University of California San Francisco, San Francisco, CA, USA. 'Department of Neuroradiology, University of Würzburg, Würzburg, Germany. ${ }^{7}$ Department of Psychology, School of Social Sciences, University of Mannheim, Mannheim, Germany. ${ }^{8}$ Department of Systems Neuroscience, University Medical Center Hamburg-Eppendorf, Hamburg, Germany. ${ }^{9}$ Department of Psychology, Humboldt University, Berlin, Germany. ${ }^{10}$ Department of Clinical Psychology and Psychotherapy, University of Cologne, Cologne, Germany. ${ }^{11}$ Department of Psychology, Institute of Clinical Psychology and Psychotherapy, Technische Universität Dresden, Dresden, Germany. ${ }^{12}$ Division of Clinical Psychology and Intervention Science, University of Basel, Basel, Switzerland. ${ }^{13}$ Department of Psychology and Psychotherapy, University of Hamburg, Hamburg, Germany. ${ }^{14}$ Neuroimaging Center (NIC) und Deutsches Resilienz-Zentrum (DRZ), Johannes Gutenberg University Medical Center, Mainz, Germany. ${ }^{15}$ Department of Psychiatry and Psychotherapy, University of Marburg, Marburg, Germany. ${ }^{16} \mathrm{Christoph}$-DornierFoundation for Clinical Psychology, Bremen, Germany. ${ }^{17}$ Department of Psychiatry and Psychotherapy, Campus Charité Mitte, Charité -

Universitätsmedizin Berlin, corporate member of the Freie Universität Berlin, Humboldt-Universität zu Berlin, and Berlin Institute of Health, Berlin, Germany. ${ }^{18}$ Department of Psychiatry, Psychosomatic Medicine and Psychotherapy, University Hospital of Frankfurt, Frankfurt, Germany. ${ }^{19}$ Department of Psychiatry and Psychotherapy, University Hospital of Münster, Münster, Germany. ${ }^{20}$ kboInn-Salzach-Hospital, Wasserburg, Germany. ${ }^{21}$ Department of Psychiatry und Psychotherapy, Ludwig Maximilians University, Munich, Germany.

${ }^{22}$ Department of Child and Adolescent Psychiatry, Psychosomatics and Psychotherapy, Center of Mental Health, University Hospital of Würzburg, Würzburg, Germany. ${ }^{23}$ Department of Psychology, Center of Mental Health, University of Würzburg, Würzburg, Germany. ${ }^{24}$ Department of Psychiatry and Psychotherapy, Medical Faculty Heinrich-Heine University, Duesseldorf, Germany. ${ }^{25}$ Center for NeuroModulation, Faculty of Medicine, University of Freiburg, Freiburg, Germany

\section{Conflict of interest}

M.G. Gottschalk, K. Domschke, J. Richter, C. Ziegler, M.A. Schiele, J. Mann, M.J. Geiger, C. Schartner, G.A. Homola, G.W. Alpers, C. Büchel, L. Fehm, T. Fydrich, A. L. Gerlach, A.T. Gloster, S. Helbig-Lang, R. Kalisch, Kircher, T. Lang, T.B. Lonsdorf, C.A. Pané-Farré, A. Ströhle, H. Weber, P. Zwanzger, M. Romanos, H.-U. Wittchen, A. Hamm, P. Pauli, A. Reif, Deckert, S. Neufang and M. Höfler declare no conflict of interest. V. Arolt has served as an Advisor for Allergan, Astra-Zeneca, Janssen, Lundbeck, Neuraxpharm, Otsuka, Organon, Servier, and Tromsdorf. He gave lectures for Janssen, Neuraxpharm, Organon, and Servier.

\section{Publisher's note}

Springer Nature remains neutral with regard to jurisdictional claims in published maps and institutional affiliations.

Supplementary Information accompanies this paper at (https://doi.org/ 10.1038/s41398-019-0415-8).

Received: 20 October 2018 Revised: 6 January 2019 Accepted: 17 January 2019

Published online: 04 February 2019

\section{References}

1. Sakurai, T. et al. Orexins and orexin receptors: a family of hypothalamic neuropeptides and $\mathrm{G}$ protein-coupled receptors that regulate feeding behavior. Cell 92, 573-585 (1998).

2. Sakurai, T. The role of orexin in motivated behaviours. Nat. Rev. Neurosci. 15 719-731 (2014).

3. Kukkonen, J. P. \& Leonard, C. S. Orexin/hypocretin receptor signalling cascades. Br. J. Pharmacol. 171, 314-331 (2014).

4. Leonard, C. S. \& Kukkonen, J. P. Orexin/hypocretin receptor signalling: a functional perspective. Br. J. Pharmacol. 171, 294-313 (2014).

5. Marcus, J. N. et al. Differential expression of orexin receptors 1 and 2 in the rat brain. J. Comp. Neurol. 435, 6-25 (2001).

6. Dresler, T. et al. Revise the revised? New dimensions of the neuroanatomical hypothesis of panic disorder. J. Neural Transm. Nienna) 120, 3-29 (2013).
7. Johnson, P. L., Molosh, A., Fitz, S. D. \& Truitt, W. A. Shekhar A. Orexin, stress, and anxiety/panic states. Prog. Brain. Res. 198, 133-161 (2012).

8. Soya, S. et al. Orexin receptor-1 in the locus coeruleus plays an important role in cue-dependent fear memory consolidation. J. Neurosci. 33, 14549-14557 (2013).

9. Sears, R. M. et al. Orexin/hypocretin system modulates amygdala-dependent threat learning through the locus coeruleus. Proc. Natl Acad. Sci. USA 110, 20260-20265 (2013).

10. Johnson, P. L. et al. Orexin 1 receptors are a novel target to modulate panic responses and the panic brain network. Physiol. Behav. 107, 733-742 (2012).

11. Johnson, P. L. et al. A key role for orexin in panic anxiety. Nat. Med. $\mathbf{1 6}$ 111-115 (2010).

12. Johnson, P. L. et al. Activation of the orexin 1 receptor is a critical component of CO2-mediated anxiety and hypertension but not bradycardia. Neuropsychopharmacology 37, 1911-1922 (2012).

13. Flores, A. et al. The hypocretin/orexin system mediates the extinction of fear memories. Neuropsychopharmacology 39, 2732-2741 (2014).

14. Salomon, R. M. et al. Diurnal variation of cerebrospinal fluid hypocretin-1 (Orexin-A) levels in control and depressed subjects. Biol. Psychiatry 54, 96-104 (2003).

15. Annerbrink, $K$ et al. Panic disorder is associated with the Val308lso polymorphism in the hypocretin receptor gene. Psychiatr. Genet. 21, 85-89 (2011).

16. Fukunaka, Y. et al. The orexin 1 receptor (HCRTR1) gene as a susceptibility gene contributing to polydipsia-hyponatremia in schizophrenia. Neuromolecular. Med. 9, 292-297 (2007).

17. Gallone, S. et al. Is HCRTR2 a genetic risk factor for Alzheimer's disease? Dement. Geriatr. Cogn. Disord. 38, 245-253 (2014).

18. Tang, S. et al. Increased plasma orexin-A levels in patients with insomnia disorder are not associated with prepro-orexin or orexin receptor gene polymorphisms. Peptides 88, 55-61 (2017).

19. Petersen, S. E. \& Posner, M. I. The attention system of the human brain: 20 years after. Annu. Rev. Neurosci. 35, 73-89 (2012).

20. Geiger, M. J., Neufang, S., Stein, D. J. \& Domschke, K. Arousal and the attentional network in panic disorder. Hum. Psychopharmacol. 29, 599-603 (2014).

21. Gloster, A. T. et al. Mechanism of action in CBT (MAC): methods of a multicenter randomized controlled trial in 369 patients with panic disorder and agoraphobia. Eur. Arch. Psychiatry Clin. Neurosci. 259, S155-S166 (2009).

22. Ho, D. E., Imai, K., King, G. \& Stuart, E. A. Matching as nonparametric preprocessing for reducing model dependence in parametric causal inference. Political Anal. 15, 199-236 (2007).

23. Ho, D. E., Imai, K., King, G. \& Stuart, E. A. Matchit: nonparametric preprocessing for parametric causal inference. J. Stat. Softw. 42, 1-28 (2011).

24. Deckert, J. et al. GLRB allelic variation associated with agoraphobic cognitions, increased startle response and fear network activation: a potential neurogenetic pathway to panic disorder. Mol. Psychiatry 22, 1431-1439 (2017).

25. Elehrs, A. \& Margraf, J. Fragebogen zu körperbezogenen Ängsten, Kognitionen und Vermeidung (AKV). (Beltz Test, Weinheim, 1993).

26. Gloster, A. T. et al. Psychological treatment for panic disorder with agoraphobia: a randomized controlled trial to examine the role of therapist-guided exposure in situ in CBT. J. Consult. Clin. Psychol. 79, 406-420 (2011).

27. Richter, J. et al. Dynamics of defensive reactivity in patients with panic disorder and agoraphobia: implications for the etiology of panic disorder. Biol. Psychiatry 72, 512-520 (2012).

28. Hamm, A. O. et al. Panic disorder with agoraphobia from a behavioral neuroscience perspective: Applying the research principles formulated by the Research Domain Criteria (RDoC) initiative. Psychophysiology 53, 312-322 (2016).

29. Thompson, M. D., Xhaard, H., Sakurai, T., Rainero, I. \& Kukkonen, J. P. OX1 and OX2 orexin/hypocretin receptor pharmacogenetics. Front. Neurosci. 8, 57 (2014).

30. Domschke, K., Deckert, J., O'Donovan, M. C. \& Glatt, S. J. Meta-analysis of COMT val158met in panic disorder: ethnic heterogeneity and gender specificity. Am. J. Med. Genet. B. Neuropsychiatr. Genet. 144B, 667-673 (2007).

31. Reif, A. et al. Meta-analysis argues for a female-specific role of MAOA-uVNTR in panic disorder in four European populations. Am. J. Med. Genet. B. Neuropsychiatr. Genet. 159B, 786-793 (2012).

32. Johren, O., Neidert, S. J., Kummer, M. \& Dominiak, P. Sexually dimorphic expression of prepro-orexin mRNA in the rat hypothalamus. Peptides $\mathbf{2 3}$, 1177-1180 (2002). 
33. Grafe, L. A., Cornfeld, A., Luz, S., Valentino, R. \& Bhatnagar, S. Orexins Mediate Sex Differences in the Stress Response and in Cognitive Flexibility. Biol. Psychiatry 81, 683-692 (2017).

34. Reinecke, A., Waldenmaier, L., Cooper, M. J. \& Harmer, C. J. Changes in automatic threat processing precede and predict clinical changes with exposurebased cognitive-behavior therapy for panic disorder. Biol. Psychiatry 73, 1064-1070 (2013).

35. Reif, A. et al. MAOA and mechanisms of panic disorder revisited: from bench to molecular psychotherapy. Mol. Psychiatry 19, 122-128 (2014).

36. Warren, R., Zgourides, G. \& Englert, M. Relationships between catastrophic cognitions and body sensations in anxiety disordered, mixed diagnosis, and normal subjects. Behav. Res. Ther. 28, 355-357 (1990).

37. Lynch, P., Bakal, D., Whitelaw, W., Fung, T. \& Rose, L. Agoraphobic avoidance and panic frequency as predictors of laboratory induced panic reactions. Behav. Res. Ther. 30, 591-596 (1992).

38. Teachman, B. A., Marker, C. D. \& Clerkin, E. M. Catastrophic misinterpretations as a predictor of symptom change during treatment for panic disorder. J. Consult. Clin. Psychol. 78, 964-973 (2010).

39. Posner, M. I. \& Rothbart, M. K. Research on attention networks as a model for the integration of psychological science. Annu. Rev. Psychol. 58, 1-23 (2007).

40. Hagan, J. J. et al. Orexin A activates locus coeruleus cell firing and increases arousal in the rat. Proc. Natl Acad. Sci. USA 96, 10911-10916 (1999).

41. Goossens, L. et al. Brainstem response to hypercapnia: a symptom provocation study into the pathophysiology of panic disorder. J. Psychopharmacol. 28 449-456 (2014).
42. Domschke, K., Stevens, S., Pfleiderer, B. \& Gerlach, A. L. Interoceptive sensitivity in anxiety and anxiety disorders: an overview and integration of neurobiological findings. Clin. Psychol. Rev. 30, 1-11 (2010).

43. Soya, $\mathrm{S}$. et al. Orexin modulates behavioral fear expression through the locus coeruleus. Nat. Commun. 8, 1606 (2017).

44. Sehlmeyer, C. et al. Neural correlates of trait anxiety in fear extinction. Psychol. Med. 41, 789-798 (2011).

45. Bouton, M. E., Mineka, S. \& Barlow, D. H. A modern learning theory perspective on the etiology of panic disorder. Psychol. Rev. 108, 4-32 (2001).

46. Helbig-Lang, S. et al. The role of safety behaviors in exposure-based treatment for panic disorder and agoraphobia: associations to symptom severity, treatment course, and outcome. J. Anxiety Disord. 28, 836-844 (2014).

47. Porter, E. \& Chambless, D. L. A systematic review of predictors and moderators of improvement in cognitive-behavioral therapy for panic disorder and agoraphobia. Clin. Psychol. Rev. 42, 179-192 (2015).

48. Schiele, M. A. \& Domschke, K. Epigenetics at the crossroads between genes, environment and resilience in anxiety disorders. Genes. Brain. Behav. 17, e12423 (2018).

49. Arendt, D. H. et al. Anxiolytic function of the orexin 2/hypocretin A receptor in the basolateral amygdala. Psychoneuroendocrinology 40, 17-26 (2014).

50. Domschke, K. Muller, D. J. \& Serretti, A. Personalized therapies in psychiatry: promises, pitfalls and perspectives. J. Neural Transm. Nienna) 122, 1-3 (2015).

51. Gottschalk, M. G. \& Domschke, K. Novel developments in genetic and epigenetic mechanisms of anxiety. Curr. Opin. Psychiatry 29, 32-38 (2016). 